\title{
A REVIEW ON ENERGY EFFICIENT JOB SCHEDULING ALGORITHMS IN GREEN CLOUD COMPUTING
}

\author{
JUHI MONDAL, PRADEEP KV, VIJAYAKUMAR V
}

Department of CSE, VIT University, Chennai, Tamil Nadu, India. Email:asha.s@vit.ac.in

Received: 19 January 2017, Revised and Accepted: 20 February 2017

\begin{abstract}
Cloud computing is the hottest topic in today's world and is being used by most of the information technology companies due to its benefits in cost saving and ease of use. It is a dynamic, scalable, and pay-per-use distributed computing model. The cloud computing is aims to give access to remote and geographically distributed resources. Scheduling the job from one data center to other data center or from one region of cloud to other region is truly a testing in cloud environment. However, when data are shifted from one data center to other data center, huge amount of carbon dioxide $\left(\mathrm{CO}_{2}\right)$ gases emits and also consumes more power. Since energy is an important issue, that is, why green cloud computing comes into the picture. Green cloud computing can be obtained by applying various techniques and algorithms, which use less power and emits less $\mathrm{CO}_{2}$ gas, that is, damaging the environment. Cloud provides many facilities to its tenants such as sharing of resources for different purposes. In cloud domain, job scheduling is one of the biggest and hypothetical problems. Numerous investigations for scheduling a job and efforts carried out in this regard because it is one of the main jobs to get the most profit. Scheduling can decrease the power consumption. So many algorithms had been proposed for this purpose, and a lot more had to be done. In the paper, intends to present a variety of energy-efficient job scheduling algorithms along with performance comparison analysis of various preexisting algorithms for scheduling jobs to provide energy efficiency in green cloud computing.
\end{abstract}

Keywords: Cloud computing, Job scheduling, Resource allocation, Efficiency, Performance, Cost.

(C) 2017 The Authors. Published by Innovare Academic Sciences Pvt Ltd. This is an open access article under the CC BY license (http://creativecommons. org/licenses/by/4. 0/) DOI: http://dx.doi.org/10.22159/ajpcr.2017.v10s1.20509

\section{INTRODUCTION}

\section{Cloud computing}

Cloud computing is an internet-based computing; the data centers are practiced using a network of remote servers that are hosted on the internet where storing, managing computer, or any local server. Its provide users to share data and resources to any computers or devices on demand. Cloud computing provides three different approaches to cloud services layer that are:

- The infrastructure is known as the IaaS service (IaaS).

- The platform is known as the PaaS service (PaaS).

- Software is known as SaaS services (SaaS).

These three services layers help in business organizations and government organizations which cut down the operational expenses.

IaaS provides services where the user has control over operating systems. IaaS provides services such as Amazon web services, IBM Bluemix, and Micorsoft Azure. It gives only the equipment and system; the client introduces or creates its own particular working frameworks, programing, and applications.

PaaS provides a platform for developing applications by the help of cloud; there is no need to install any platform if the user is using it on its own machine. PaaS service such as Windows azure and Google App Engine. It provides the working environment along with equipment, and system are given, the client introduces or builds up their own applications using a particular programing languages.

SaaS for running existing applications such as Instagram, Facebook, and Google Apps, where users do not handle the installation of any software on their personal computers. It is an exceedingly software package as a service model, a pre-made application, aboard any needed programing, operating framework, equipment, and system are given.

\section{Green cloud computing}

Cloud contain thousands of data centers to fulfil the demands of customers online, on time and because of that data centers is span in an area of hundred to thousand feet. Very huge amount of power is required to run these servers. Hence, green cloud computing [10] is not only picturized to achieve the efficient processing and utilized the computing infrastructure, but it is also for minimizing the energy consumption that is effecting the environment in a larger amount. Green cloud computing is extremely important for ensuring that the coming year of cloud computing must be sustainable and eco-friendly. Green cloud works

on large scale companies such as Google and Amazon IBM cloud because its enables user to get services anywhere. Green cloud computing are the on-demand services because its shares big resource pools that user can buy on request or requirement. In the paper, Wadhwa and Verma [11] have reviewed about the various researchers and their strategies to make cloud computing more energy efficient, to reduce the carbon emission from the environment and found virtualization can help in utilization of resources in clouds.

Scheduling

Clients among themselves share resources and distributes and schedules their jobs within the cloud, it has become a challenge to schedule these jobs. Hence, job scheduling is a hottest topic in cloud computing. The scheduling algorithms belonging to distributed systems mainly aim to partition the load and assign sub loads to processors to attain their most utilization whereas minimizing the entire load execution time. During this, the jobs need to be moved or scheduled from one data center to other, which will emit a lot of carbon dioxide $\left(\mathrm{CO}_{2}\right)$ and also consumes more power. Hence, it is needed to provide a flexible and reliable systems to avail green cloud computing.

Scheduling can be done at either cloud level or user level. Cloud level job scheduling is ordered at client side and framework level. At client, it manages issues raised by administrator related to procurement 
between suppliers and clients. The framework level, it handles asset administration inside data center. Data center is the one which is a collection of more number of physical machines.

Scheduling is one of the crucial and important activity needed to be performed in a cloud computing environment. Scheduling improves the efficiency of cloud workloads. This is the job of maximizing profits, throughput, and make-span. Scheduling algorithms in cloud computing is to utilize the resources meanwhile balancing the load between the resources so that, the resources execute in a minimal execution time [14]. There are many scheduling algorithms in cloud computing. Scheduling algorithms is done to obtain high performance, utilize the resources, and managing the load between the resources. Examples of scheduling algorithms are first come first serve (FCFS), round robin algorithm, shortest job first (SJF), min-min algorithm, max-max algorithm, and so on. Green scheduling algorithms [10] that based neural predictors can saved up to $70 \%$ of power savings. These algorithms enable us to cut down the data center energy costs and lead us to strong competitive cloud computing environment.

In a single data center, thousands of servers will be running, and user demands for the requests to be executed consecutively or concurrently. During this, if server is busy then one has to notice for an alternative server, which is ready and can fulfil the batch of incoming jobs. This is why job/task scheduling is an important issue, which greatly affects the performance of cloud services. Job scheduling can also be categorized based on load balancing, temperature based, and energy efficiency.

\section{Scheduling classifications}

\section{Preemptive and non-preemptive scheduling}

- The scheduling algorithms which falls in the above-discussed categories, permits interruption of job in the middle of execution, and resources allocated is taken off from the current job and will be allocated to other job just arrived which has the highest priority than current one. Hence, we call them as preemptive.

- If suppose the resources are not taken off from the current job which is running until the execution completes, then we call them as nonpreemptive.

\section{Dynamic and static scheduling algorithm}

- Dynamic: System will not have any information about the arrival of job, time required to execute, and resources to be allocated are not known well in advance. Now system has to take care about all these things and respond as per demand.

- Static: In this, it involves less runtime overhead as it involves prior fetching of the required data and pipelining of different task execution stages, i.e., well in advance, it knows about the arrival time of jobs, allocation of resources such as central processing unit (CPU), memory usage, and time required for execution.

\section{Immediate and batch scheduling}

- Batch scheduling: Controlling unattended background program execution of jobs and execution of non-interactive jobs is called as batch processing, sometimes called as workload automation. Queue is a data structure used to implement batch. In this, all similar jobs are grouped together and submitted as a batch for processing, which in turn system will consider and starts executing one after the other automatically, so called workload automation.

- Immediate: Here, jobs are scheduled as soon as they arrive, and no need to wait for any resources such as CPU, memory, and so on.

\section{Decentralized and centralized scheduling}

- Decentralized: It is well-suited for those organizations, whose business applications are running on cloud and provisioning the required resources from cloud. As the name suggest, it is decentralized, so there is no centralized system, where it manages and maintains the status of jobs which are queued locally and never suffers from bottleneck problems.

- Centralized: Here, we will be having a system which is located centrally, and will be monitored, controlled, and maintain the status of each job which is queued locally. All the decisions are made globally and will be centralized. It has many benefits as easy to implement; more efficient and provides better control over resources. However, very much lacks in scalability, fault tolerance and efficient performance, due to the centralization.

\section{Energy efficient}

- The goal of energy efficiency is to reduce the energy consumption of the organization to achieve or provide users with good products and services. Reducing energy reduces the cost, and it can be seen as to reduce the greenhouse gases. As today's world, users are more on cloud computing where number of tasks are executed at a time, power of consumption is reduced, and $\mathrm{CO}_{2}$ emission is increasing in an alarming rate so energy efficient scheduling algorithm is required in cloud computing. The remainder of the paper contains the following: Section II gives the motivation of the research, Section III contains the objective, Section IV contains the literature survey, and Section $\mathrm{V}$ presents the conclusion.

\section{MOTIVATION}

Cloud computing is an important model in the field of information technology (IT), in which huge number of task are performed and requirement of resources, on demand are more because of that servers becomes busier. In data center, where all servers' physical resources are available, machines are consuming more power and heat which is effecting the environmental conditions. This is due to companies give more focus to the higher availability than on energy efficiency. The main purpose of green cloud computing is to reduce the energy that is consumed by the data centers. Technique used for reducing the power consumption and make algorithm energy efficient is job scheduling. Many works have been done in the field of job scheduling for increasing the throughput, performance but a lot more have to be done on energy efficiency.

\section{OBJECTIVE}

In today's world, user are more interested in cloud computing because its saving their cost and time. As its usage increasing, started effecting the environment by emitting the heat and $\mathrm{CO}_{2}$. The main purpose of this paper is to review, all the available job scheduling algorithms that are made for energy efficiency and in what way these algorithms have an impact in producing the less $\mathrm{CO}_{2}$, which emits emissions from the environment.

\section{LITERATURE SURVEY}

In this section, we discuss about the various job scheduling algorithm along with analysis by considering some important parameters such as load balancing and soon. It is intended to present a variety of energy-efficient job scheduling algorithms along with performance comparison analysis of various preexisting algorithms for scheduling jobs to provide energy efficiency in green cloud computing. Scheduling in cloud is generally comprised three stages: Resource discovery and filtering - resources in network and their related status information are collected. Resource selection target resource is chosen based on various parameters. Job submission - job is submitted to the chosen resource.

Various advancements have been made toward different calculations for designating, scheduling, and scaling the assets productively in the cloud. The target of scheduling is execution upgrade and enhancing the nature of administration alongside keeping up the productivity and decency among the employments and decrease the execution cost.

The following job scheduling algorithms are currently exist in the cloud and summarize these algorithms. 
A job scheduling algorithm based on preemption priority In the paper, Kaur and Midha [1] have proposed an energy efficient job scheduling algorithm that focuses on the preemptive part as well as it calculates the energy consumption for scheduling the jobs on the cloud computing servers. The main aim of the author is to minimize the less $\mathrm{CO}_{2}$ gases and maximize the resources but according to the suitability of servers. In the paper, the computing server is selected on the basis of that satisfies the minimum resource requirement on a job. Resources are allocated by the method of best allocation scheme that saves energy and by creating a balance between the power consumption and work load on the servers.

\section{Energy efficient scheduling and traffic load balancing}

In the paper, Kliazovich et al. [2] have proposed scheduling algorithm for optimizing the power consumption of data center equipment that provides load efficient allocation of network traffic QoS to improve operations in cloud applications by delaying congestion-related packet loss. The author has used green cloud simulator for the experimental results on algorithms. The proposed algorithm shows that the energy consumption of ordinary paper management in the data center does not increase.

\section{Priority-based job scheduling algorithm}

In the paper, Kaur and Singh [4] have proposed an algorithm that is two-fold mechanism. The first phase calculates the priority of the job that is given according to the specialized attributes in the job and then, it is sorted by the calculated priority. Second-phase computes the time that each task will require to execute. The paper presents an algorithm that schedule the job in an order that apart from calculating the priority of tasks it will calculate the expected executable time of different task on different servers. The author uses CloudSim simulator for the experimental results. Furthermore, the priority is calculated under different attributes such as user level, expected priority, waiting time, and based on the formula each attributes of priority can be achieved. They use the concept of standardization that will merge the given attributes into a single attribute. The algorithm achieved a scheduling that is based on priority parameter. The algorithm provides a decent level of load balancing and cost minimization.

\section{SJF scheduling}

In the paper, Bakely and Hefny's approach [5] is to propose the job scheduling algorithm to minimize the energy in a cloud computing which is totally based on enhancing the green scheduler which performs the work load consolidation on a minimum server in green cloud computing by the help of executing task which have minimum arrival time. This paper presents a data center scheduling approach that helps in reducing the power consuming and achieves balance between the two factors energy efficient and performance. The author has used green cloud simulator for the experimental results for simulation work they use domain name system scheme for minimizing energy consumption. The applied algorithm reduces the energy in server up to $33.99 \%$, in switch energy $49.65 \%$ and data center $38.04 \%$. Its reduce energy consumption in all the components of cloud computing.

\section{Greedy scheduling of tasks with time constraints}

In the paper, Dong et al.'s [6] aim was to find the optimal task scheduling scheme to minimize the task response time and energy consumed by the data center server. The authors use the most efficient server first scheme where the server with the highest computing capacity will provide a lower energy expenditure per processed job then the optimization problem can be interpreted as a greedy assignment scheme. It is believed that the central scheduler classifies the servers based on their energy efficiency, and it assigns tasks to the most energy efficient servers first. The author uses Matlab simulation. The proposed scheme saves energy of long task response time within the maximum constraint and server-related energy consumption. Its save more than 70 times the average data center.
Energy-saving green scheduling algorithm in green cloud computing

In the paper, Truong and Inoguchi [7] propose a green scheduling algorithm, the workload will focus on the server, and then turn it off. They use neural network-based predictors to save energy in cloud computing. Predictors can predict future load requirements based on historical requirements. The green scheduling algorithm determines which server should be turned off/on. It will turn on when the workload is heavy and off when the workload is small. In the paper, author uses CloudSim and GridSim toolkit for experimental results. They performed the simulation using four different running modes on two traces that were NASA and the Clarknet load traces. The running modes are normal mode, optimal green mode, prediction green mode, prediction plus additional servers. From the results, they found that prediction plus $20 \%$ additional servers was the best configuration to ensure service levels. Its provides a $46.3 \%$ reduction in power with a drop rate of $0.03 \%$ at Clarknet and a $46.7 \%$ reduction in power and a drop rate of $0.12 \%$ at NASA.

\section{Workflow job scheduling in green cloud computing}

In the paper, Cao and Zhu [8] have proposed an efficient scheduling algorithm for scientific workflow that is multi-step heuristics workflow scheduling algorithm named energy aware resource efficient workflow scheduling in the deadline constraints. It addresses various objectives that include guaranteed QoS, reduce energy, and $\mathrm{CO}_{2}$ emissions for energy saving and eco-friendly data center. They proposed the algorithm which is designed to meet response time requirements and minimize virtual machine to reduce power consumption. The author has used Java-based CloudSim toolkit for green cloud computing infrastructure and evaluated the scheduling algorithms. The simulation results of their proposed algorithm show the energy consumption from e-ECT to dynamic voltage and frequency scaling (DVFS) is reduced by $11 \%$, the energy consumption from federal wage system to body weight support is reduced by $12 \%$, and an average of $30 \%$ energy savings and resource utilization can be achieved.

\section{Energy-aware real-time and non-real-time scheduling}

In the paper, Reddy and Chandan [12] have put forward one algorithm deals with real-time and non-real-time Task. They used three processors the first two processor used real-time tasks using earliest deadline first and earliest deadline late scheduling algorithm and also realtime tasks its uses a Stand by sparing technique and for non-real-time tasks are scheduled using FCFS scheduling algorithm. For simulation authors used Matlab. The algorithm that they have proposed for both tasks had conserved energy up to $58 \%$ when compared to any power management and up to $4 \%$ energy when compared to existing system.

\section{Efficient energy the hybrid and DVFS-enabled cloud computing workflow tasks}

In the paper, Tang et al. [13] have proposed an DVFS enables energy efficient workflow task scheduling in which the energy-aware methods distribute parallel applications to the optimal processor and process them to reduce the energy consumption on appropriate time slots and meet the performance required to perform the workflow within a deadline. The evaluation is done between the DEWTS has two heuristics algorithms, HEFT and EES. They merge processors that much to find out the processor which are relatively inefficient and assign the tasks to the appropriate timeslot and expand its execution time. The experiment was carried out by the use of CloudSim simulator. The experimental results show that the EES saves up to $44 \%$ of energy when CCR is 0.5 and $37 \%$ less energy when CCR is 2. Furthermore, when the CCR to 2 DEWTS, the energy saving up to $46 \%$.

CONCLUSION

In the paper, we reviewed and investigated a variety of existing energy efficient job scheduling algorithms in green cloud computing. There are many parameters that can be mentioned as a factor of scheduling problem that can be considered - such as load balancing, throughput, 
service cost, and so forth. Some of the results that shows that these algorithms have proved to be energy efficient such as in the workflow job scheduling algorithm have saved up to $30 \%$ of energy. Real-time and non-real-time tasks in energy-aware scheduling algorithms save up to $58 \%$ of energy. In most of the algorithm, authors have used tools kit for finding the experimental results, they were CloudSim and Matlab toolkits. In SJF, scheduling and e-STAB the experimental was carried out by the use of Green cloud simulator. There is a certain feature that should be considered as topics of research to produce more accurate and improved algorithms and a lot more work had to be done in future to provide eco-friendly cloud computing.

\section{REFERENCES}

1. Kaur G, Midha S. A pre-emptive priority based job scheduling algorithm in green cloud computing. In: $6^{\text {th }}$ International ConferenceClous System and Big Data Engineering (Confluence); 2016.

2. Kliazovich D, Arzo ST, Granelli F, Bouvry P, Khan SU. E-STAB: Energy-efficient scheduling for cloud computing applications with traffic load balancing. In: IEEE International Conference on Green Computing and Communications and IEEE Internet of Things and IEEE Cyber, Physical and Social Computing; 2013.

3. Kumar K, Hans A, Sharma A, Singh N. Towards the various cloud computing scheduling concerns: A review and quot. In: International Conference on Innovative Applications of Computational Intelligence on Power, Energy and Controls with their Impact on Humanity
(CIPECH14) 28 and 29 November; 2014

4. Kaur P, Singh P. Priority based scheduling algorithm with fast task completion rate in cloud. Int J Sci Technol Res 2013;2(11):

5. Bakely AH, Hefny HA. Using shortest job first scheduling in greenloud computing. Int J Adv Res 2015;4:348-54.

6. Dong Z, Liu N, Rojas-Cessa R, et al. Greedy scheduling of tasks withtime constraint for energy efficient cloud computing data centers. J Cloud Comput 2015;4:5.

7. Truong TV, Inoguchi YS. Performance evaluation of a green scheduling algorithm for energy savings in cloud computing. Jpn Adv Inst Sci Technol

8. Cao F, Zhu MM. Energy Efficient Workflow Job Scheduling for Green Cloud. IEEE International Conference on Green Computing and Communications; 2013.

9. Shimpy ER, Sidhu J. Different scheduling algorithms in different cloud environment. Int J Adv Res Comput Commun Eng 2014;4(9):8003-6.

10. Gayathri B. Green cloud computing (seiscon 2012); 2012. p. 27-9.

11. Wadhwa B, Verma A. Energy Saving Approaches for Green clouComputing: A Review. IEEE; 2014.

12. Reddy SP, Chandan HK. Energy Aware Scheduling of Real Time and Non-Real Time Tasks on cloud Processor Green Cloud Computing. IEEE; 2014.

13. Tang Z, Cheng Z, Li K. An Efficient Energy Scheduling Algorithm for Workflow Tasks in Hybrids and DVFS Enabled cloud Environment. IEEE; 2014

14. Shimpy ER, Sidhu J. Different scheduling algorithms in different cloud environment. Int J Adv Res Comput Commun Eng 2014;4(9):8003-6. 